\title{
Current practice in benzodiazepine receptor agonists deprescribing on acute geriatric wards: a cohort study
}

François-Xavier Sibille $e^{1,2,3^{*}} \mathbb{0}$, Anne Spinewine ${ }^{3,4}$, Lorène Zerah ${ }^{3}$, Laurentine Maljean ${ }^{4}$,

Didier Schoevaerdts ${ }^{1,2}$ and Marie de Saint-Hubert ${ }^{1,2,5}$

\begin{abstract}
Background: Benzodiazepine receptor agonist (BZRA) use is highly prevalent in hospitalised older people although these drugs are associated with numerous and serious adverse events. Deprescribing can reduce risks associated with chronic BZRA use. The aim of this study was to measure the prevalence of, and factors associated with, BZRA deprescribing in acute geriatric units.
\end{abstract}

Methods: During a one-year period, this multicentre retrospective study included patients aged $\geq 70$ years, hospitalised in acute geriatric units, and using $\geq 1$ BZRA on admission. BZRA deprescribing at discharge was defined as: $\geq 25 \%$ decrease in lorazepam-equivalent admission dose; discontinuation of all BZRAs; or cessation of a rescue prescription at discharge. BZRA cessation was defined as discontinuation of all BZRAs at discharge. We identified social, medical, geriatric and medication factors associated with BZRA deprescribing using logistic regression.

Results: In total, 561 patients were included (mean age: $85.3 \pm 5.9$ years, $70 \%$ of women). BZRA deprescribing occurred in 240 (42.8\%), including 85 with BZRA cessation (15.2\%). Deprescribing occurred more frequently in patients with a BZRA-related adverse event on admission or during hospital stay (odds ratio (OR) $4.5 ; 95 \%$ confidence interval $[2.6 ; 7.9])$, with an antidepressant $(1.6[1.1 ; 2.4])$ and a higher lorazepam-equivalent dosage on admission (OR $1.2[1 ; 1.4])$, and less frequently in patients with antipsychotic drug (OR $0.5[0.3 ; 0.8])$. BZRA cessation was more likely in patients with a BZRA-related adverse event (OR $2.2[1.2 ; 4.3])$ and a lower lorazepam-equivalent dosage on admission (OR $0.5[0.3 ; 0.6])$.

Conclusions: During hospitalisation in the acute geriatric units of our hospital, BZRA deprescribing occurred in $42.8 \%$ of the patients. Identification of an BZRA-related adverse event by the treating physician appears to be a major factor: this reactive deprescribing accounted for $74 \%$ of cases in our study. Further prospective studies are needed to measure long-term persistence of in-hospital deprescribing and encourage proactive management.

Keywords: Benzodiazepines, Deprescribing, Frail elderly, Hospitalization

*Correspondence: francois-xavier.sibille@uclouvain.be

${ }^{3}$ Clinical Pharmacy Research Group, Louvain Drug Research Institute,

Université Catholique de Louvain, Avenue Mounier, 72 bte B1.72.02,

1200 Brussels, Belgium

Full list of author information is available at the end of the article

\section{Introduction}

Benzodiazepine receptor agonists (BZRA), including benzodiazepines and $\mathrm{z}$-drugs, are the most prescribed psychotropic drugs in older people [1,2], with prescription rates ranging from $7 \%$ to $25 \%$ in elderly persons living in the community $[3,4]$, to $52 \%$ in long-term-care residents [5], and $32.6 \%$ in hospitalised patients [6]. 
BZRAs may be appropriate in a few circumstances, such as seizure disorders or ethanol withdrawal. However, they are mainly used in the treatment of sleep disorders and anxiety [7]. For these indications, they are often inappropriate because of their moderate and short-term efficacy and their numerous and serious adverse events, such as delirium, falls and fractures [8-10]. Although, the American Geriatric Society [11], the START-STOPP criteria [12], and the FORTA list [13] recommend that BZRAs should be avoided in all older people, and certainly in those with dementia, risk of delirium, or risk of falls.

Deprescribing can be defined as the process of withdrawal of an inappropriate medication under the supervision of a healthcare professional with the goal of managing polypharmacy and improving outcomes [14]. There is a growing body of evidence that BZRA deprescribing can reduce the risk of harm associated with chronic BZRA use, with no worsening of, or even improvement in, sleep quality, and reduction in anxiety or depression [15-18]. The success rates reported in older people vary according to the definition of deprescribing (discontinuation only or dose reduction), the setting in which the effects of deprescribing are measured, the nature of the interventions, the type of analysis, and the follow-up duration. For example, BZRA deprescribing success rates range from $27 \%$ to $80 \%$ in older people living in the community [19-21], and from $33 \%$ to $66 \%$ in nursing-home settings [5, 22].

Despite this available literature, several research gaps remain. Firstly, few studies have explored BZRA deprescribing during acute hospitalisation. Two studies targeted geriatric units, but were monocentre, had only limited sample sizes, and mental and cognitive disorders were exclusion criteria [23, 24]. Moreover, although there is a global awareness of the barriers and facilitators of successful general deprescribing at the physician (existence of recommendations, concerns about adverse withdrawal effects, medication initially prescribed by another physician ... ) and patient (trust in the treating physician, experience of burden from medications, increased involvement ... ) levels [25-28], specific factors related to BZRA deprescribing have been less explored. Finally, although geriatricians are well aware of the risks of BZRA use in frail hospitalised older patients, little is known about how much they modify BZRA prescription during usual care in acute geriatric units (AGU).

The main aim of the present study was to measure the prevalence of, and factors associated with, BZRA deprescribing in acute geriatric wards. Secondary aims were to analyse BZRA cessation and associated factors; switch to another BZRA molecule; switch to another sedative molecule.

\section{Methodology}

\section{Study design, study setting and eligibility criteria}

We retrospectively screened the medical records of all patients aged 70 years or older, hospitalised during 2018 in three acute geriatric units (AGUs) in the region of Namur, Belgium. The three AGUs had a combined total of 81 beds and 1435 hospitalisations during 2018, comprising 1285 different patients. The AGUs receive patients with acute pathologies and offer interdisciplinary care (physiotherapist, occupational therapist, speech therapist, and dietician). One AGU is in a teaching hospital and has a clinical pharmacist in the interdisciplinary team.

Patients were included if they were using BZRAs $(\geq 1$ BZRA at admission, on a regular basis or as needed). Patients who died during their hospital stay or for whom there was no record of their discharge treatment were excluded because BZRA use at discharge could not be analysed. For patients hospitalised several times during 2018, only the first admission was analysed.

\section{Outcomes}

The main outcome measure was BZRA deprescribing at discharge, defined as: $\geq 25 \%$ decrease in lorazepamequivalent admission dose; discontinuation of all BZRA; or cessation of a rescue BZRA prescription [5]. Secondary outcomes were BZRA cessation at discharge defined as cessation of all BZRAs (subset of BZRA deprescribing), BZRA switch defined as a change of BZRA molecule between admission and discharge with or without a change in lorazepam-equivalent dose, and switch to another sedative molecule defined as new prescriptions of trazodone or mirtazapine at discharge.

\section{Data collection and variables}

All data (demographic, clinical and medication) were extracted from the patient electronic records, including emergency department or other original ward discharge letters, geriatric ward discharge letters and medication lists, geriatric mobile unit reports, and laboratory values. Data extraction was performed by two researchers (a geriatrician, FXS, and a clinical pharmacist with clinical practice in geriatrics, LM) using a written protocol. For a selection of 30 files, extraction was performed independently by both researchers and discrepancies were discussed and clarifications added to the standard operating procedure.

We used the Cumulative Illness Rating Scale-Geriatric (CIRS-G) [29] to estimate comorbidity burden (theoretical maximum score $=56$ ). We recorded the presence or absence of: delirium, according to the clinical judgment of the treating geriatrician; cognitive decline, if a diagnosis of dementia had been established or if the Mini 
Mental State Evaluation was less than 24 points, outside a context of delirium. Discharge to another ward was defined as discharge to a short stay in a nursing home or to another hospital ward.

On admission and at discharge we collected the number of prescribed medications; the number, molecules and dosages of BZRA (Appendix 1); and the number and molecules of other psychotropic drugs (N02A, N03A, N04, N05A, N05C, N06A-C-D). BZRA dosages were converted into lorazepam-equivalent doses using a conversion table [30, 31], so that molecules could be compared at baseline and between admission and discharge. Polypharmacy was defined as regular use of 5 or more drugs [32] and excessive polypharmacy as use of 10 or more drugs [33]. Multi-BZRA users were defined as users of $\geq 2$ BZRA on a regular basis and/or for rescue use. Psychotropic polypharmacy was defined as concomitant use of $\geq 3$ central nervous system (CNS)-acting drugs [11]. BZRA-related adverse event was defined as any adverse event present on admission or during hospitalization, explicitly related to BZRA use, and documented as such in the electronic medical record, based on the clinical judgment of the treating team. These adverse events were falls, dizziness, drowsiness and delirium.

\section{Statistical analysis}

Data are presented as mean (standard deviation [SD]) or median (first and third quartile [Q1; Q3]) for continuous variables, and number (percentage) for categorical variables. Normality was assessed using the Kolmogorov-Smirnov test and a graphical representation of the distribution. The Mann-Whitney U test or Student $t$ test was used for continuous variables and Pearson's chi-squared test or Fisher's exact test for categorical variables.

Binary logistic regression models were developed to assess independent variables associated with BZRA deprescribing and cessation, and adjusted odds ratios (OR) were calculated with their 95\% confidence intervals (95CI). Factors potentially associated with BZRA deprescribing were selected through literature review [34-38], included demographic and administrative data (including length of stay), factors related to possible indications for BZRA prescription, comorbidities of relevance when considering BZRA use in older people, and adverse events related to BZRA use (Table 1). Variables with a $p$-value $<.10$ in univariate analysis (Appendix 4) were eligible for the multivariable model. Correlation between variables was assessed using a principal component analysis. The choice between two correlated variables was made based on their respective clinical relevance. We also included other variables that we expected to be associated with the likelihood of BZRA deprescribing. There were no missing data. All tests were 2 -sided, and a $p<.05$ was considered statistically significant.

All analyses were performed using R software v.4.0.3 (R Foundation for Statistical Computing, Vienna, Austria).

\section{Results \\ Population description}

Among the 1285 patients admitted in AGUs, 561 were using BZRA and were included in the study (Figure 1). Patient characteristics are shown in Table 1. The mean patient age was $85.3 \pm 5.9$ years; $70 \%$ were women and $73 \%$ lived in the community. The level of comorbidities was high (median CIRS-G 22 points) [39]. Anxiety and sleep disorders were mentioned for $16.4 \%$ and $8.7 \%$ of patients, respectively. More than half of the patients had cognitive decline and one third had delirium on admission. Polypharmacy and excessive polypharmacy were highly prevalent: $91.6 \%$ of the patients were taking 5 or more daily medications and $44.6 \% 10$ or more. Psychotropic polypharmacy affected $41.2 \%$ of the patients (Table 1). Median length of stay was 15 days with (interquartile range: 11-22 days). Patient characteristics by AGU are presented in Appendix 2.

\section{BZRA deprescribing and associated factors}

At discharge, BZRAs had been deprescribed in 240 patients (42.8\%): 85 patients (15.2\% of all included patients) had stopped taking any BZRA (i.e. BZRA cessation), 194 (34.6\%) had had a reduction in lorazepamequivalent dosage of at least $25 \%$, and $49(8.7 \%)$ had stopped taking one or more rescue BZRAs. In some patients two of the three criteria were met.

In the multivariate analysis (Table 2), a BZRA-related adverse event during the hospital stay (OR 4.5 [2.6 -7.9]), a higher lorazepam-equivalent dose (OR 1.2 [1-1.4]) and use of an antidepressant $(1.6[1.1 ; 2.4])$ were significantly associated with BZRA deprescribing. In the opposite, patients using also an antipsychotic drug on admission had a significantly lower rate of BZRA deprescribing (OR $0.5[0.3-0.8])$.

\section{BZRA cessation and associated factors}

At discharge, 85 patients (15.2\%) were no longer prescribed any BZRA. In the multivariate analysis (Table 2), BZRA-related adverse event during the hospital stay (OR $2.2[1.2 ; 4.3])$ was significantly associated with BZRA cessation. Patients with higher lorazepam-equivalent dosage on admission experienced significantly less BZRA cessation (OR $0.5[0.3 ; 0.6])$.

\section{Switch of BZRA molecule}

The most frequent BZRA molecules on admission were lorazepam (30.2\%) and alprazolam (14.7\%) for 
Table 1 Population characteristics

\begin{tabular}{|c|c|c|c|c|}
\hline & $\begin{array}{l}\text { BZRA users } \\
N=561\end{array}$ & $\begin{array}{l}\text { BZRA deprescribing } \\
N=240\end{array}$ & $\begin{array}{l}\text { BZRA continuation } \\
N=321\end{array}$ & $p$ value $^{a}$ \\
\hline \multicolumn{5}{|l|}{ Demographic data } \\
\hline Age (years), mean, SD & $85.3 ; 5.9$ & $84.8 ; 5.9$ & $85.7 ; 5.8$ & .066 \\
\hline Female, $n(\%)$ & $392(69.9)$ & $168(70.0)$ & $224(69.8)$ & .999 \\
\hline \multicolumn{5}{|l|}{ Place of residence, n (\%) } \\
\hline Home & $412(73.4)$ & $165(68.7)$ & $247(76.9)$ & \\
\hline Nursing home & $149(26.6)$ & $75(31.3)$ & $74(23.1)$ & .015 \\
\hline \multicolumn{5}{|l|}{ Comorbidities } \\
\hline CIRS-G, median; Q1-Q3 & $22 ; 18-25$ & $22 ; 19-26$ & $21 ; 17-25$ & $<.001$ \\
\hline Anxiety, n (\%) & $92(16.4)$ & $44(18.3)$ & $48(15.0)$ & .340 \\
\hline Sleep disorder, n (\%) & $49(8.7)$ & $22(9.2)$ & $27(8.4)$ & .871 \\
\hline Restless leg, n (\%) & $16(2.9)$ & $7(2.9)$ & $9(2.8)$ & .999 \\
\hline Depression, n (\%) & $149(26.6)$ & $71(29.6)$ & $78(24.3)$ & .192 \\
\hline Bipolar or psychotic disorder, n (\%) & $16(2.9)$ & $7(2.9)$ & $9(2.8)$ & .999 \\
\hline Extrapyramidal syndrome, n (\%) & $58(10.3)$ & $21(8.8)$ & $37(11.5)$ & .353 \\
\hline Previous fracture, n (\%) & $201(35.8)$ & $93(38.8)$ & $108(33.6)$ & .247 \\
\hline Fall at admission, $n(\%)$ & $274(48.8)$ & $115(47.9)$ & $159(49.5)$ & .769 \\
\hline Current fracture, n (\%) & $85(15.2)$ & $31(12.9)$ & $54(16.8)$ & .247 \\
\hline COPD, n (\%) & $82(14.6)$ & $36(15.0)$ & $46(14.3)$ & .919 \\
\hline Delirium, n (\%) & $188(33.5)$ & $95(39.6)$ & $93(29.0)$ & .017 \\
\hline Cognitive decline, n (\%) & $316(56.3)$ & $148(61.7)$ & $168(52.3)$ & .034 \\
\hline \multicolumn{5}{|l|}{ Administrative data } \\
\hline \multicolumn{5}{|l|}{ Admission, n (\%) } \\
\hline Emergency & $110(19.6)$ & $52(21.7)$ & $58(18.1)$ & \\
\hline Transfer & $65(11.6)$ & $34(14.2)$ & $31(9.7)$ & .099 \\
\hline Direct to AGU & $386(68.8)$ & $154(64.2)$ & $232(72.3)$ & \\
\hline Length of stay (days), median; Q1-Q3 & $15 ; 11-22$ & $16 ; 12-23$ & $15 ; 11-22$ & .181 \\
\hline \multicolumn{5}{|l|}{ Discharge destination, $\mathrm{n}(\%)$} \\
\hline Home & $277(49.4)$ & $107(44.6)$ & $170(53.0)$ & \\
\hline Nursing home & $200(35.7)$ & $102(42.5)$ & $98(30.5)$ & .040 \\
\hline Another ward & $84(14.9)$ & $31(12.9)$ & $53(16.5)$ & \\
\hline \multicolumn{5}{|l|}{ Medication at admission } \\
\hline Total medications, median; Q1-Q3 & $9 ; 7-12$ & $9 ; 7-12$ & $9 ; 7-11$ & .174 \\
\hline Polypharmacy, n (\%) & $514(91.6)$ & $218(90.8)$ & $296(92.2)$ & .668 \\
\hline Excessive polypharmacy, n (\%) & $250(44.6)$ & $116(48.3)$ & $134(41.7)$ & .142 \\
\hline BZRA dose, median; Q1-Q3 & $1.0 ; 1.0-2.0$ & $1.33 ; 0.9-2.5$ & $1.0 ; 1.0-2.0$ & .023 \\
\hline Multi-BZRA users, n (\%) & $112(20.0)$ & $73(30.4)$ & $39(12.1)$ & $<.001$ \\
\hline Psychotropic polpharmacy, n (\%) & $231(41.2)$ & $119(49.6)$ & $112(34.9)$ & $<.001$ \\
\hline Antipsychotic users, $n(\%)$ & $116(20.7)$ & $43(17.9)$ & $73(22.7)$ & .270 \\
\hline Antidepressant users, $\mathrm{n}(\%)$ & $273(48.7)$ & $135(56.3)$ & $138(43.0)$ & .002 \\
\hline Trazodone & $59(10.5)$ & $32(13.3)$ & $27(8.4)$ & .054 \\
\hline Mirtazapine & $45(8.0)$ & $21(8.8)$ & $24(7.5)$ & .586 \\
\hline \multicolumn{5}{|l|}{ Miscellaneous } \\
\hline Palliative status, $n(\%)$ & $42(7.5)$ & $20(8.3)$ & $22(6.9)$ & .619 \\
\hline Intervention of a clinical pharmacist, $\mathrm{n}(\%)$ & $202(36.0)$ & $103(42.9)$ & $99(30.8)$ & .002 \\
\hline $\begin{array}{l}\text { Identification of a BZRA-related adverse event by the treating physi- } \\
\text { cian, } \mathrm{n}(\%)\end{array}$ & $88(15.7)$ & $65(27.1)$ & $23(7.2)$ & $<.001$ \\
\hline BZRA switch between admission and discharge, $\mathrm{n}(\%)$ & $65(11.6)$ & $26(10.8)$ & $39(12.1)$ & .870 \\
\hline
\end{tabular}

SD standard deviation, Q1 first quartile, Q3 third quartile, CIRS-G Cumulative Illness Rating Scale-Geriatric, COPD Chronic Obstructive Pulmonary Disease, AGU acute geriatric unit

${ }^{a} p$ value of difference between deprescribing and continuation using Mann-Whitney $\mathrm{U}$ test or Student $\mathrm{t}$ test for continuous variables and Pearson's chi-squared test or Fisher's exact test for categorical variables 


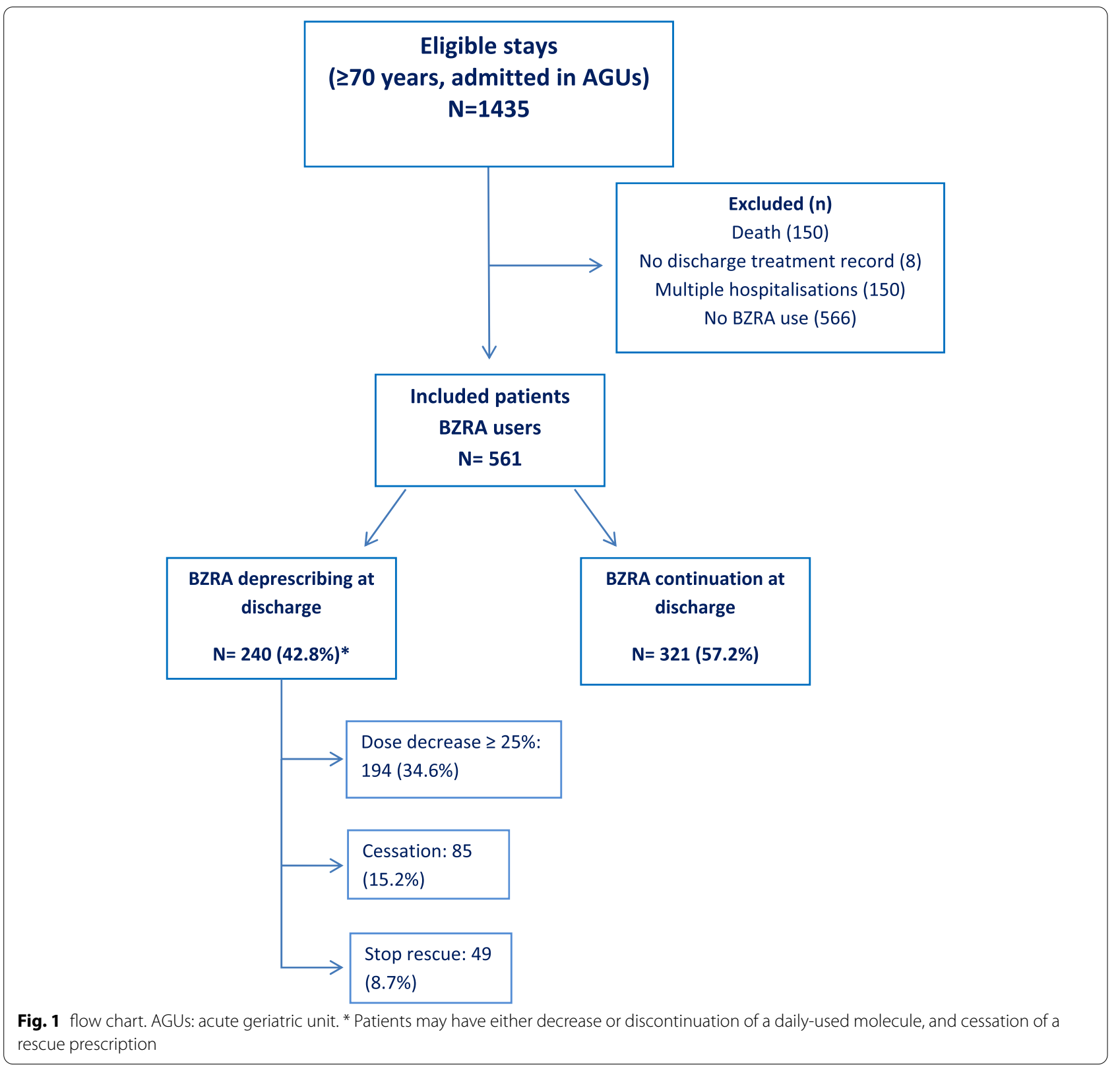

regular users, and alprazolam (31.4\%) and prazepam (27.9\%) for rescue users (Appendix 3). Regarding the half-lives of the molecules, $70.8 \%$ of the patients were taking a medium-acting BZRA. At discharge, the most frequent molecules were lorazepam and alprazolam for both regular and rescue use. BZRA switch occurred in $11.6 \%$ of patients. There was a significant shift from long- to medium-acting molecules at discharge $(76.7 \%$ for regular use and $89.3 \%$ for rescue use, $p=0.025$ and $p<0.001$, respectively) (Appendix 3 ). There were no differences between groups with or without BZRA deprescribing.

\section{Switch to another sedative molecule}

Fourteen (6.0\%) of the patients who had BZRA deprescribing were given new prescriptions for trazodone, and $6(1.8 \%)$ of the patients with BZRA continuation $(p=0.011)$. Mirtazapine was newly prescribed to 27 patients, distributed evenly between the groups with and without deprescribing (data not shown). Eight patients in the BRZA cessation group (9.4\%) were given new prescriptions for trazodone, and $12(2.5 \%)$ in the patients with BRZA continuation $(p=0.006)$. There were no differences in new mirtazapine prescriptions in the patients with BZRA cessation and BZRA continuation. 
Table 2 Factors associated with BZRA deprescribing and BZRA cessation: multivariate analysis

\begin{tabular}{|c|c|c|c|c|}
\hline & \multicolumn{2}{|c|}{ BZRA deprescribing } & \multicolumn{2}{|c|}{ BZRA cessation } \\
\hline & OR $[95 \mathrm{Cl}]$ & $p$ value & OR [95Cl] & $p$ value \\
\hline \multicolumn{5}{|l|}{ Demographic data } \\
\hline $\mathrm{Age}^{\mathrm{a}}$ & $1[0.9 ; 1.0]$ & .218 & $1[0.9 ; 1.0]$ & .085 \\
\hline \multicolumn{5}{|l|}{ Comorbidities } \\
\hline CIRS-G ${ }^{a}$ & $1[1 ; 1.1]$ & .110 & $1[1.0 ; 1.1]$ & .489 \\
\hline Depression & $1.2[0.8 ; 1.9]$ & .365 & $0.9[0.5 ; 1.7]$ & .809 \\
\hline Delirium & $1.2[0.8 ; 1.8]$ & .457 & $1.2[0.7 ; 2.1]$ & .474 \\
\hline Cognitive decline & $1.5[1 ; 2.3]$ & .066 & $1[0.6 ; 1.8]$ & .971 \\
\hline Anxiety & $1.2[0.7 ; 2.1]$ & .437 & $1[0.5 ; 2]$ & .991 \\
\hline Sleep disorder & $1.1[0.6 ; 2.1]$ & .780 & $1.7[0.7 ; 3.7]$ & .191 \\
\hline \multicolumn{5}{|l|}{ Administrative data } \\
\hline Admission directly to $\mathrm{AGU}$ & 1 & & 1 & \\
\hline Admission through emergency room & $1.1[0.7 ; 1.9]$ & .651 & $0.7[0.3 ; 1.3]$ & .256 \\
\hline Admission from another ward & $1.5[0.8 ; 2.6]$ & .193 & $1[0.4 ; 2]$ & .931 \\
\hline Length of stay ${ }^{a}$ & $1[1 ; 1]$ & .215 & $1[1 ; 1]$ & .116 \\
\hline Living in a nursing home & $1.3[0.7 ; 2.3]$ & .592 & $1.4[0.6 ; 3.2]$ & .527 \\
\hline Discharge to home & 1 & & 1 & \\
\hline Discharge to a nursing home & $1.5[0.8 ; 2.7]$ & .172 & $1.4[0.6 ; 2.9]$ & .387 \\
\hline Discharge to another ward & $0.8[0.4 ; 1.4]$ & .404 & $0.5[0.2 ; 1.1]$ & .124 \\
\hline \multicolumn{5}{|l|}{ Medications at admission } \\
\hline BZRA dosage ${ }^{a}$ & $1.2[1.0 ; 1.4]$ & .020 & $0.5[0.4 ; 0.6]$ & $<.001$ \\
\hline Antipsychotic user & $0.5[0.3 ; 0.8]$ & .008 & $0.7[0.3 ; 1.3]$ & .236 \\
\hline Antidepressant user & $1.6[1.1 ; 2.4]$ & .027 & $1.4[0.8 ; 2.5]$ & .243 \\
\hline \multicolumn{5}{|l|}{ Miscellaneous } \\
\hline Intervention of a clinical pharmacist & $1.5[0.9 ; 2.3]$ & .162 & $0.8[0.4 ; 1.5]$ & .421 \\
\hline $\begin{array}{l}\text { Identification of a BZRA-related adverse event by the } \\
\text { treating physician }\end{array}$ & $4.5[2.6 ; 8]$ & $<.001$ & $2.4[1.2 ; 4.5]$ & .012 \\
\hline
\end{tabular}

CIRS-G Cumulative Illness Rating Scale-Geriatric, OR Odds ratio, 95Cl 95\% confidence interval

${ }^{\text {a }}$ Numeric variables

\section{Discussion}

At discharge, respectively $42.8 \%$ and $15.2 \%$ of older patients hospitalized in 3 Belgian AGUs experienced BZRA deprescribing and cessation. The presence of a BZRA-related adverse event on admission or during hospitalisation was the main predictor of both outcomes. This deprescribing in response to an adverse clinical trigger is called reactive deprescribing and accounted for $74 \%$ of deprescribing occurrences in our study. During hospitalisation, there was also a significant switch from long- to medium-acting molecules at discharge. Respectively $12.0 \%$ and $14.1 \%$ of the patients with BZRA deprescribing or cessation were prescribed another sedative molecule. To the best of our knowledge, this is the first complete description of BZRA deprescribing (including dose tapering and psychotropic drugs co-prescription) during hospitalisation in AGUs.
Our rate of deprescribing is lower than the $53 \%$ of patients with a fall in a geriatric rehabilitation context in Australia [40]. Our rate of cessation (15.2\%) was also lower than the rate $(20.8 \%)$ of the control group in the pilot study that evaluated the efficacy of a patient- educational brochure [41]. Similar to our data, an English observational study found that reactive deprescribing accounted for $80 \%$ of all drugs combined deprescribing activities during acute hospitalisation [42].

Higher BZRA doses significantly increased the probability of BZRA deprescribing but reduced the probability of BZRA cessation. For any increase of one unit lorazepam-equivalent, there was $20 \%$ more chance of BZRA deprescribing and $50 \%$ less chance of BZRA cessation. We can hypothesise that this contrasting finding may be the result of the limited length of stay of our patients. Most BZRA tapering methods recommend schedules of at least one month [41], but three quarters of our patients 
had a length of stay of less than 22 days. Use of an antidepressant was associated with a higher probability of BZRA deprescribing, in line with what was suggested by a recent Cochrane Database of Systematic Review [43]. Use of an antipsychotic was associated with a lower probability of BZRA deprescribing, which has not, to our knowledge, been described previously. We can hypothesize that patients using antipsychotic drugs may have more severe behavioural symptoms.

We expected the intervention of a clinical pharmacist to influence BZRA deprescribing and cessation. Indeed, several trials using pharmacist involvement have shown a positive impact on BZRA deprescribing [44-46]. A recent systematic review suggested that pharmacist involvement as part of a multidisciplinary team might improve the quality of prescribing in older inpatients [47]. Despite greater deprescribing in the univariate analysis with intervention of a clinical pharmacist, this was not confirmed in the multivariable analysis, possibly due to insufficient power and because part of this effect was captured by the BZRA-related adverse events, which a clinical pharmacist may help to identify.

One may be concerned that BZRA deprescribing would just reflect a switch to another sedative medication, such as trazodone or mirtazapine [48, 49]. Indeed, psychotropic polypharmacy was already high in our population [6] and is also a subject of concern in older people [11]. It is encouraging to see that this situation did not occur frequently, as another sedative molecule was prescribed in only $12.0 \%$ and $14.1 \%$ of BZRA deprescribing or cessation cases.

In patients without BZRA deprescribing, BZRA molecule was switched in $11.6 \%$ of cases, with a significant switch from long- to medium-acting molecules with a better pharmacokinetic profile in older people. This change in profile may be viewed as an improvement [50].

One aspect regarding our population deserves closer examination: anxiety and sleep disorders were rarely mentioned in the medical records despite high general comorbidity rates [51]. This low rate of anxiety and sleep disorders suggests that BZRAs may not be indicated in these patients [5], and/or that the indication for BZRA is often not documented. Identifying the indication for a medication is the first step in developing a deprescribing algorithm [52], and is an important challenge and an area that needs to be improved to promote deprescribing.

Hospitalisation may be questioned as the best setting for deprescribing of such medication. Indeed, in line of the results of the present study, it represents an opportunity to initiate deprescribing for several reasons [42, 48]: 1) Patients can benefit from close monitoring of withdrawal symptoms; 2) frequent diagnoses on admission, such as falls or delirium, may be related to BZRA use before hospitalisation; 3) AGUs provide patientcentred approach, multidisciplinary teams and geriatrician insight, all known to improve deprescribing rates [5, 18, 34, 53]. However, hospitalised patients may experience more anxiety and sleep disorders [51], and have less confidence in healthcare professionals at the hospital than in their general practitioner or usual nursing team. The acute medical problem leading to hospitalisation may also detract the attention of the professionals away from performing BZRA deprescribing. Recent data inform on key determinants and behavioural change techniques to improve deprescribing in the hospital setting [54]. For instance, interventions should facilitate shared deprescribing decision making.

The strength of this study is that it provides a reallife picture of BZRA management in the AGUs. No specific deprescribing program was implemented and patients received usual geriatric care. We also included cognitively impaired patients who are often excluded from deprescribing trials [21, 55], and provide a context of other psychotropic drugs use. The main limitations of the study are its retrospective design limiting the availability and quality of the data. For example, no systematic screening for delirium was performed, so that its prevalence may have been underestimated, especially that of hypoactive delirium. Secondly, because of the lack of follow-up, we do not know whether BZRA deprescribing was maintained or whether BRZAs were started again after hospital discharge $[48,56]$. We also have no information on the duration of BZRA use, alcohol consumption, and deprescribing failure or refusal during the hospital stay, all factors that could potentially influence BZRA deprescribing rates.

In conclusion, this retrospective study showed that BZRA deprescribing was initiated in $42.8 \%$ of patients hospitalised in 3 Belgian acute geriatric wards. Identification of a BZRA-related adverse event by the treating physician, higher lorazepam-equivalent dosage and use of an antidepressant were associated with BZRA deprescribing and cessation. Use of an antipsychotic drug was associated with a lower rate of BZRA deprescribing. Routine clinical work should better document indications for BZRA intake and identify opportunities to proactive deprescribing. Future studies should investigate mid-term persistence of BZRA deprescribing and evaluate patient- and healthcare professionalcentred interventions to optimise BZRA deprescribing in hospital. 


\section{Appendix 1}

Tables 3

Table 3 BZRA lorazepam-equivalent dosage and duration of action

\begin{tabular}{llll}
\hline INN & ATC code & $\begin{array}{l}\text { Equivalent } \\
\text { dosage }(\mathbf{m g})\end{array}$ & Duration of action \\
\hline diazepam & N05BA01 & 10 & Long-acting \\
alprazolam & N05BA12 & 0.5 & Medium-acting \\
bromazepam & N05BA08 & 4.5 & Medium-acting \\
brotizolam & N05CD09 & 0.25 & Medium-acting \\
clobazam & N05BA09 & 10 & Long-acting \\
clonazepam & N03AE01 & 0.5 & Long-acting \\
clorazepate & N05BA05 & 10 & Long-acting \\
clotiazepam & N05BA21 & 5 & Medium-acting \\
cloxazolam & N05BA22 & 1 & Long-acting \\
flunitrazepam & N05CD03 & 0.5 & Long-acting \\
flurazepam & N05CD01 & 15 & I Long-acting \\
ethyl loflazepate & N05BA18 & 1 & Long-acting \\
loprazolam & N05CD11 & 0.5 & Medium-acting \\
lorazepam & N05BA06 & 1 & Medium-acting \\
lormetazepam & N05CD06 & 1 & Medium-acting \\
midazolam & N05CD08 & 7.5 & Short-acting \\
nitrazepam & N05CD02 & 5 & Long-acting \\
nordazepam & N05BA16 & 2.5 & Long-acting \\
oxazepam & N05BA04 & 15 & Medium-acting \\
prazepam & N05BA11 & 20 & Long-acting \\
triazolam & N05CD05 & 0.125 & Short-acting \\
zolpidem & N05CF02 & 10 & Short-acting \\
zopiclone & N05CF01 & 7.5 & Short-acting \\
\hline
\end{tabular}

INN international non-proprietary name, ATC Anatomic Therapeutic Chemical classification

\section{Appendix 2}

Table 4

Table 4 Patients' characteristics by AGU

\begin{tabular}{|c|c|c|c|}
\hline & $\begin{array}{l}\text { AGU } 1 \\
(n=172)\end{array}$ & $\begin{array}{l}\text { AGU } 2 \\
(n=215)\end{array}$ & $\begin{array}{l}\text { AGU } 3 \\
(n=174)\end{array}$ \\
\hline \multicolumn{4}{|l|}{ Demographic data } \\
\hline Age (years): mean; SD & $86.7 ; 5.6$ & $84.4 ; 5.7$ & $85.1 ; 6.2$ \\
\hline Female & $123(71.5 \%)$ & $143(66.5 \%)$ & $126(72.4 \%)$ \\
\hline \multicolumn{4}{|l|}{ Comorbidities } \\
\hline CIRS-G: median; Q1-Q3 & $20 ; 17-23$ & $24 ; 20-28$ & $20.5 ; 17-24.75$ \\
\hline Anxiety & $10(5.8 \%)$ & $59(27.4 \%)$ & $23(13.2 \%)$ \\
\hline Sleep disorder & $5(2.9 \%)$ & $26(12.1 \%)$ & $18(10.3 \%)$ \\
\hline Restless leg syndrome & $1(0.6 \%)$ & $11(5.1 \%)$ & $4(2.3 \%)$ \\
\hline Depression & $33(19.2 \%)$ & $63(29.3 \%)$ & $53(30.5 \%)$ \\
\hline Extrapyramidal syndrome & $13(7.6 \%)$ & $25(11.6 \%)$ & $20(11.5 \%)$ \\
\hline COPD & $23(13.4 \%)$ & $34(15.8 \%)$ & $25(14.4 \%)$ \\
\hline Cognitive decline & $129(75.0 \%)$ & $83(38.6 \%)$ & $104(59.8 \%)$ \\
\hline Delirium & $55(32.0 \%)$ & $76(35.3 \%)$ & $57(32.8 \%)$ \\
\hline Fall at admission & $77(44.8 \%)$ & $112(52.1 \%)$ & $85(48.9 \%)$ \\
\hline Current fracture & $22(12.8 \%)$ & $30(14.0 \%)$ & $33(19.0 \%)$ \\
\hline Previous fracture & $60(34.9 \%)$ & $83(38.6 \%)$ & $58(33.3 \%)$ \\
\hline Psychiatric disorder & $3(1.7 \%)$ & $5(2.3 \%)$ & $8(4.6 \%)$ \\
\hline \multicolumn{4}{|l|}{ Administrative data } \\
\hline \multicolumn{4}{|l|}{ Place of residence: } \\
\hline Home & $122(70.9 \%)$ & $169(78.6 \%)$ & $121(69.5 \%)$ \\
\hline Nursing home & $50(29.1 \%)$ & $46(21.4 \%)$ & $53(30.5 \%)$ \\
\hline $\begin{array}{l}\text { Length of stay (days): median; } \\
\text { Q1-Q3 }\end{array}$ & $15 ; 11.75-20$ & $15 ; 11-21.5$ & $16 ; 11-26$ \\
\hline \multicolumn{4}{|l|}{ Admission route: } \\
\hline Emergency & $146(84.9 \%)$ & $116(54.0 \%)$ & $124(71.3 \%)$ \\
\hline Direct & $15(8.7 \%)$ & $70(32.6 \%)$ & $25(14.4 \%)$ \\
\hline Transfer & $11(6.4 \%)$ & $29(13.5 \%)$ & $25(14.4 \%)$ \\
\hline \multicolumn{4}{|l|}{ Discharge destination: } \\
\hline Home & $88(51.2 \%)$ & $108(50.2 \%)$ & $81(46.6 \%)$ \\
\hline Nursing home & $61(35.5 \%)$ & $68(31.6 \%)$ & $71(40.8 \%)$ \\
\hline Another ward & $23(13.4 \%)$ & $39(18.1 \%)$ & $22(12.6)$ \\
\hline \multicolumn{4}{|l|}{ Medications at admission } \\
\hline $\begin{array}{l}\text { Total medications: median; } \\
\text { Q1-Q3 }\end{array}$ & $8.5 ; 6-10.25$ & $10 ; 8-13$ & $8 ; 6-11$ \\
\hline Polypharmacy & $149(86.6 \%)$ & $206(95.8 \%)$ & $159(91.4 \%)$ \\
\hline Excessive polypharmacy & $63(36.6 \%)$ & $122(56.7 \%)$ & $65(37.4 \%)$ \\
\hline BZRA dose: median; Q1-Q3 & $1.3 ; 1-2.5$ & $1 ; 0.95-2$ & $1 ; 0.85-2$ \\
\hline Multi-BZRA users & $29(16.9 \%)$ & $51(23.7 \%)$ & $32(1.8 \%)$ \\
\hline Psychotropic drug users & $119(69.2 \%)$ & $157(73.0 \%)$ & $123(70.7 \%)$ \\
\hline Psychotropic polypharmacy & $69(40.1 \%)$ & $97(45.1 \%)$ & $65(37.4 \%)$ \\
\hline Antipsychotic users & $39(22.7 \%)$ & $43(20.0 \%)$ & $34(19.5 \%)$ \\
\hline Antidepressant users & $76(44.2 \%)$ & $118(54.9 \%)$ & $79(45.4 \%)$ \\
\hline Trazodone users & $13(7.6 \%)$ & $30(14.0 \%)$ & $16(9.2 \%)$ \\
\hline \multicolumn{4}{|l|}{ Miscellaneous } \\
\hline Palliative status & $10(5.8 \%)$ & $8(3.7 \%)$ & $24(13.8 \%)$ \\
\hline $\begin{array}{l}\text { Intervention of a clinical } \\
\text { pharmacist }\end{array}$ & $0(0.0 \%)$ & $202(94.0 \%)$ & $0(0.0 \%)$ \\
\hline $\begin{array}{l}\text { Identification of a BZRA-related } \\
\text { adverse event by the treating } \\
\text { physician }\end{array}$ & $11(6.4 \%)$ & $51(23.7 \%)$ & $26(14.9 \%)$ \\
\hline $\begin{array}{l}\text { BZRA switch between admission } \\
\text { and discharge }\end{array}$ & $11(6.4 \%)$ & $22(10.2 \%)$ & $32(18.4 \%)$ \\
\hline
\end{tabular}

SD standard deviation, Q1 first quartile, Q3 third quartile, CIRS-G Cumulative Illness Rating Scale-Geriatric, COPD Chronic Obstructive Pulmonary Disease, AGU acute geriatric unit 


\section{Appendix 3}

Table 5

Table 5 BZRA molecules and categories of duration of action

\begin{tabular}{|c|c|c|c|}
\hline & Admission & Discharge & $p$ value \\
\hline \multicolumn{4}{|l|}{ Regular use } \\
\hline \multicolumn{4}{|c|}{ BZRA frequent molecules } \\
\hline Alprazolam & $101[14.7 \%]$ & 88 [15.6\%] & $<.001$ \\
\hline Bromazepam & $63[9.1 \%]$ & $46[8.2 \%]$ & \\
\hline Lorazepam & 208 [30.2\%] & $251[44.6 \%]$ & \\
\hline Lormetazepam & $73[10.6 \%]$ & 65 [11.5\%] & \\
\hline Zolpidem & 71 [10.3\%] & 55 [9.8\%] & \\
\hline \multicolumn{4}{|l|}{ BZRA categories } \\
\hline Short-acting & 91 [13.2\%] & 75 [12.2\%] & .025 \\
\hline Medium-acting & $488[70.8 \%]$ & $470[76.7 \%]$ & \\
\hline Long-acting & 110 [16.0\%] & $68[11.1 \%]$ & \\
\hline \multicolumn{4}{|l|}{ Rescue use } \\
\hline \multicolumn{4}{|c|}{ BZRA frequent molecules } \\
\hline Alprazolam & 27 [31.4\%] & 18 [23.7\%] & .040 \\
\hline Bromazepam & $4[4.7 \%]$ & $4[5.3 \%]$ & \\
\hline Lorazepam & 15 [17.4\%] & $43[56.6 \%]$ & \\
\hline prazepam & 24 [27.9\%] & 3 [3.9\%] & \\
\hline \multicolumn{4}{|l|}{ BZRA categories } \\
\hline Short-acting & $5[5.9 \%]$ & 2 [2.7\%] & $<.001$ \\
\hline Medium-acting & $51[60.0 \%]$ & 67 [89.3\%] & \\
\hline Long-acting & $29[34.1 \%]$ & 6 [8.0\%] & \\
\hline
\end{tabular}

\section{Appendix 4}

Table 6

Table 6 Factors associated with BZRA deprescribing: Univariate analysis

\begin{tabular}{|c|c|c|}
\hline & OR $[95 \mathrm{Cl}]$ & $p$ value \\
\hline \multicolumn{3}{|l|}{ Demographic data } \\
\hline Age & $1[0.9 ; 1]$ & .080 \\
\hline Female & $1[0.7 ; 1.4]$ & .927 \\
\hline \multicolumn{3}{|l|}{ Place of residence } \\
\hline Home & 1 & \\
\hline Nursing home & $1.5[1 ; 2.2]$ & .030 \\
\hline \multicolumn{3}{|l|}{ Comorbidities } \\
\hline CIRS-G & $1.1[1 ; 1.1]$ & .001 \\
\hline Anxiety & $1.3[0.9 ; 2.1]$ & .194 \\
\hline Sleep disorder & $1.2[0.6 ; 2.1]$ & .636 \\
\hline Restless leg syndrome & $1.1[0.4 ; 3]$ & .867 \\
\hline Depression & $1.3[0.9 ; 2]$ & .161 \\
\hline Bipolar or psychotic disorder & $1.1[0.4 ; 3]$ & .867 \\
\hline Extrapyramidal syndrome & $0.7[0.4 ; 1.1]$ & .147 \\
\hline Previous fracture & $1.3[0.9 ; 1.8]$ & .201 \\
\hline Fall at admission & $1[0.7 ; 1.3]$ & .825 \\
\hline Current fracture & $0.8[0.5 ; 1.2]$ & .288 \\
\hline COPD & $1[0.6 ; 1.7]$ & .847 \\
\hline Delirium & $1.7[1.2 ; 2.4]$ & .009 \\
\hline Cognitive decline & $1.5[1.1 ; 2.1]$ & .028 \\
\hline \multicolumn{3}{|l|}{ Administrative data } \\
\hline \multicolumn{3}{|l|}{ Admission } \\
\hline Direct to AGU & 1 & \\
\hline Emergency & $1.3[0.9 ; 2]$ & .214 \\
\hline Transfer & $1.7[1 ; 2.9]$ & .033 \\
\hline Length of stay & $1[1 ; 1]$ & .126 \\
\hline \multicolumn{3}{|l|}{ Discharge destination } \\
\hline Home & 1 & \\
\hline Nursing home & $1.6[1.1 ; 2.3]$ & .007 \\
\hline Another ward & $0.9[0.5 ; 1.4]$ & .776 \\
\hline \multicolumn{3}{|l|}{ Medication at admission } \\
\hline Total medications & $1[1 ; 1.1]$ & .218 \\
\hline Polypharmacy & $0.8[0.4 ; 1.5]$ & .460 \\
\hline Excessive polypharmacy & $1.3[1 ; 1.9]$ & .094 \\
\hline BZRA dose & $1.3[1.1 ; 1.5]$ & .003 \\
\hline Multi-BZRA users & $3.3[2.2 ; 5.2]$ & $<.001$ \\
\hline Psychotropic polpharmacy & $2[1.4 ; 2.8]$ & $<.001$ \\
\hline Antipsychotic users & $0.8[0.5 ; 1.2]$ & .164 \\
\hline Antidepressant users & $1.8[1.3 ; 2.5]$ & .002 \\
\hline Trazodone & $1.8[1 ; 3]$ & .033 \\
\hline Mirtazapine & $1.2[0.7 ; 2.3]$ & .483 \\
\hline \multicolumn{3}{|l|}{ Miscellaneous } \\
\hline Palliative status & $1.2[0.6 ; 2.2]$ & .630 \\
\hline Intervention of a clinical pharmacist & $1.8[1.2 ; 2.5]$ & .003 \\
\hline $\begin{array}{l}\text { Identification of a BZRA-related adverse event by the } \\
\text { treating physician }\end{array}$ & $5.1[3.1 ; 8.6]$ & $<.001$ \\
\hline BZRA switch between admission and discharge & $0.9[0.5 ; 1.6]$ & .766 \\
\hline
\end{tabular}




\section{Abbreviations}

AGU: Acute geriatric wards; BZRA: Benzodiazepine receptor agonists; CIRS-G: Cumulative Illness Rating Scale-Geriatric; CNS: Central nervous system; COPD: Chronic Obstructive Pulmonary Disease; OR: Odd's ratio; Q1; Q3: First and third quartiles; SD: Standard deviation; 95Cl: 95\% confidence intervalle.

\section{Acknowledgements}

The first author would like to thank Maxime Regnier (CHU UCL Namur) for his support in the statistical analysis.

\section{Authors' contributions}

Conceptualization: FXS MdSH, AS, LZ; data collection: FXS and LM; statistical analysis: FXS; writing —original draft preparation: FXS, MdSH; writing-review and editing: FXS, LZ, DS, AS, MdSH. All authors have read and agreed to the published version of the manuscript.

\section{Funding}

The main investigator (FXS) was funded by UCLouvain (Fonds Spéciaux de recherche) for his PhD program and, since 2020, is a Clinical Master Specialist Applicant for a Ph.D. of the Fonds de la Recherche Scientifique - FNRS. The funder had no role in study design, data collection, data analysis, data interpretation, or writing of the report.

\section{Availability of data and materials}

The datasets used and/or analysed during the current study are available from the corresponding author on reasonable request.

\section{Declarations}

\section{Ethics approval and consent to participate}

All methods were performed in accordance with the declaration of Helsinki. This study received the approval of the local ethical committees of the $\mathrm{CHU} \cup \mathrm{CL}$ Namur (Ethic committee of CHU UCL Namur (Dinant, OM1 10): 01-2019; Ethic committee CHU UCL Namur (Godinne, OM039): B039201940248; Ethic committee of CHU UCL Namur (Sainte-Elisabeth, OM070): 13-19). The informed consent of patients was not required because of the retrospective nature of the study.

\section{Consent for publication}

Not applicable

\section{Competing interests}

None

\section{Author details}

${ }^{1}$ Department of Geriatric Medicine, CHU Dinant Godinne UCL Namur, Avenue Dr Gaston Therasse, 1, 5530 Yvoir, Belgium. ${ }^{2}$ Institute of Health and Society, Université Catholique de Louvain, Clos Chapelle aux Champs, 30 Bte B1. 30.13, 1200 Brussels, Belgium. ${ }^{3}$ Clinical Pharmacy Research Group, Louvain Drug Research Institute, Université Catholique de Louvain, Avenue Mounier, 72 bte B1.72.02, 1200 Brussels, Belgium. ${ }^{4}$ Department of Pharmacy, CHU Dinant Godinne UCL Namur, Avenue Dr Gaston Therasse, 1, 5530 Yvoir, Belgium. ${ }^{5}$ NARILIS, Namur, Belgium.

Received: 1 July 2021 Accepted: 20 December 2021 Published online: 01 February 2022

\section{References}

1. Huerta C, Abbing-Karahagopian V, Requena G, Oliva B, Alvarez Y, Gardarsdottir $\mathrm{H}$, et al. Exposure to benzodiazepines (anxiolytics, hypnotics and related drugs) in seven European electronic healthcare databases: a cross-national descriptive study from the PROTECT-EU Project. Pharmacoepidemiol Drug Saf. 2016;25(Suppl 1):56-65.

2. Petrovic M, Spatharakis G, Conroy S, Van Maeles G, Moulias S. Prevalence of sedative drug use in geriatric in-patients: a multi-centre study. Acta Clin Belg. 2006;61(3):119-26.

3. Johnell K, Fastbom J. The use of benzodiazpines and related drugs amongst older people in Sweden: associated factors and concomitant use of other psychotropics. Int J Geriatr Psychiatry. 2009;24(7):731-8.
4. Grossi CM, Richardson K, Fox C, Maidment I, Steel N, Loke YK, et al. Anticholinergic and benzodiazepine medication use and risk of incident dementia: a UK cohort study. BMC Geriatr. 2019;19(1):276.

5. Evrard P, Henrard S, Foulon V, Spinewine A. Benzodiazepine Use and Deprescribing in Belgian Nursing Homes: Results from the COME-ON Study. J Am Geriatr Soc. 2020;n/a(n/a):2768-77.

6. Beuscart JB, Ficheur G, Miqueu M, Luyckx M, Perichon R, Puisieux F, et al. Co-prescriptions of psychotropic drugs to older patients in a general hospital. Eur Geriatr Med. 2017;8(1):84-9.

7. Petrovic M, Mariman A, Warie H, Afschrift M, Pevernagie D. Is there a rationale for prescription of benzodiazepines in the elderly? Review of the literature. Acta Clin Belg. 2003;58(1):27-36.

8. Sithamparanathan AS K, Leung L. Adverse effects of benzodiazepine use in elderly people: A meta-analysis. Asian J Gerontol Geriatr. 2012;7:107-11.

9. Glass J, Lanctôt KL, Herrmann N, Sproule BA, Busto UE. Sedative hypnotics in older people with insomnia: meta-analysis of risks and benefits. BMJ (Clinical research ed). 2005;331(7526):1169.

10. Tseng LY, Huang ST, Peng LN, Chen LK, Hsiao FY. Benzodiazepines, z-Hypnotics, and Risk of Dementia: Special Considerations of Half-Lives and Concomitant Use. Neurotherapeutics. 2020;17(1):156-64.

11. Panel BtAGSBCUE. American Geriatrics Society 2019 Updated AGS Beers Criteria ${ }^{\circledR}$ for Potentially Inappropriate Medication Use in Older Adults. J Am Geriatr Soc. 2019;67(4):674-94.

12. Gallagher P, O'Mahony D. STOPP (Screening Tool of Older Persons' potentially inappropriate Prescriptions): application to acutely ill elderly patients and comparison with Beers' criteria. Age Ageing. 2008;37(6):673-9.

13. Pazan F, Weiss C, Wehling M. The FORTA (Fit fOR The Aged) List 2018: Third Version of a Validated Clinical Tool for Improved Drug Treatment in Older People. Drugs Aging. 2019;36(5):481-4.

14. Reeve E, Gnjidic D, Long J, Hilmer S. A systematic review of the emerging de fi nition of 'deprescribing' with network analysis: implications for future research and clinical practice. Br J Clin Pharmacol. 2015;80(6):1254-68.

15. Ng BJ, Le Couteur DG, Hilmer SN. Deprescribing Benzodiazepines in Older Patients: Impact of Interventions Targeting Physicians, Pharmacists, and Patients. Drugs Aging. 2018;35(6):493-521.

16. Lähteenmäki R, Neuvonen PJ, Puustinen J, Vahlberg T, Partinen M, Räihä I, et al. Withdrawal from long-term use of zopiclone, zolpidem and temazepam may improve perceived sleep and quality of life in older adults with primary insomnia. Basic Clin Pharmacol Toxicol. 2019;124(3):330-40.

17. Puustinen J, Lähteenmäki R, Polo-Kantola P, Salo P, Vahlberg T, Lyles A, et al. Effect of withdrawal from long-term use of temazepam, zopiclone or zolpidem as hypnotic agents on cognition in older adults. Eur J Clin Pharmacol. 2014;70(3):319-29.

18. Paquin AM, Zimmerman K, Rudolph JL. Risk versus risk: a review of benzodiazepine reduction in older adults. Expert Opin Drug Saf. 2014;13(7):919-34

19. Curran HV, Collins R, Fletcher S, Kee SC, Woods B, Iliffe S. Older adults and withdrawal from benzodiazepine hypnotics in general practice: effects on cognitive function, sleep, mood and quality of life. Psychol Med. 2003:33(7):1223-37

20. Salonoja M, Salminen M, Aarnio P, Vahlberg T, Kivela SL. One-time counselling decreases the use of benzodiazepines and related drugs among community-dwelling older persons. Age Ageing. 2010;39(3):313-9.

21. Tannenbaum C, Martin P, Tamblyn R, Benedetti A, Ahmed S. Reduction of inappropriate benzodiazepine prescriptions among older adults through direct patient education: the EMPOWER cluster randomized trial. JAMA Internal Med. 2014;174(6):890-8.

22. Bourgeois J, Elseviers MM, Van Bortel L, Petrovic M, Vander Stichele RH. Feasibility of discontinuing chronic benzodiazepine use in nursing home residents: a pilot study. Eur J Clin Pharmacol. 2014;70(10):1251-60.

23. Petrovic M, Pevernagie D, Van Den Noortgate N, Mariman A, Michielsen W, Afschrift M. A programme for short-term withdrawal from benzodiazepines in geriatric hospital inpatients: success rate and effect on subjective sleep quality. Int J Geriatr Psychiatry. 1999;14(9):754-60.

24. Petrovic M, Pevernagie D, Mariman A, Van Maele G, Afschrift M. Fast withdrawal from benzodiazepines in geriatric inpatients: a randomised double-blind, placebo-controlled trial. Eur J Clin Pharmacol. 2002:57(11):759-64 
25. Djatche L, Lee S, Singer D, Hegarty SE, Lombardi M, Maio V. How confident are physicians in deprescribing for the elderly and what barriers prevent deprescribing? J Clin Pharmacy Therapeutics. 2018;43(4):550-5.

26. Rozsnyai Z, Jungo KT, Reeve E, Poortvliet RKE, Rodondi N, Gussekloo J, et al. What do older adults with multimorbidity and polypharmacy think about deprescribing? The LESS study - a primary care-based survey. BMC Geriatr. 2020;20(1):435.

27. Palagyi A, Keay L, Harper J, Potter J, Lindley RI. Barricades and brickwalls--a qualitative study exploring perceptions of medication use and deprescribing in long-term care. BMC Geriatr. 2016;16:15.

28. Reeve E, To J, Hendrix I, Shakib S, Roberts MS, Wiese MD. Patient barriers to and enablers of deprescribing: a systematic review. Drugs Aging. 2013;30(10):793-807.

29. Miller MD, Paradis CF, Houck PR, Mazumdar S, Stack JA, Rifai AH, et al. Rating chronic medical illness burden in geropsychiatric practice and research: Application of the Cumulative Illness Rating Scale. Psychiatry Res. 1992;41(3):237-48.

30. Holleck JL, Merchant N, Gunderson CG. Symptom-Triggered Therapy for Alcohol Withdrawal Syndrome: a Systematic Review and Meta-analysis of Randomized Controlled Trials. J Gen Intern Med. 2019;34(6):1018-24.

31. Rao SK, Mahulikar A, Ibrahim M, Shah A, Seraji-Bozorgzad N, Mohamed W. Inadequate benzodiazepine dosing may result in progression to refractory and non-convulsive status epilepticus. Epileptic Disord. 2018;20(4):265-9.

32. Jensen LD, Andersen $O$, Hallin M, Petersen J. Potentially inappropriate medication related to weakness in older acute medical patients. Int J Clin Pharm. 2014;36(3):570-80.

33. Díez-Manglano J, Giménez-López M, Garcés-Horna V, Sevil-Puras M, Castellar-Otín E, González-García P, et al. Excessive polypharmacy and survival in polypathological patients. Eur J Clin Pharmacol. 2015;71(6):733-9.

34. Joester J, Vogler CM, Chang K, Hilmer SN. Hypnosedative use and predictors of successful withdrawal in new patients attending a falls clinic: a retrospective, cohort study. Drugs Aging. 2010;27(11):915-24.

35. Aoki T, Yamamoto Y, Ikenoue T, Fukuhara S. Factors associated with patient preferences towards deprescribing: a survey of adult patients on prescribed medications. Int J Clin Pharm. 2019;41(2):531-7.

36. Alvarenga JM, Loyola Filho Al, Firmo JO, Lima-Costa MF, Uchoa E. A population based study on health conditions associated with the use of benzodiazepines among older adults (The Bambuí Health and Aging Study). Cad Saude Publica. 2009;25(3):605-12.

37. Allary A, Proulx-Tremblay V, Bélanger C, Hudon C, Marchand A, O'Connor K, et al. Psychological predictors of benzodiazepine discontinuation among older adults: Results from the PASSE 60. Addict Behav. 2020;102:106195.

38. Couderc AL, Bailly-Agaledes C, Camalet J, Capriz-Ribière F, Gary A, Robert $P$, et al. Adaptations of psychotropic drugs in patients aged 75 years and older in a departement of geriatric internal medecine: report of 100 cases. Geriatr Psychol Neuropsychiatr Vieil. 2011;9(2):163-70.

39. Salvi F, Miller MD, Grilli A, Giorgi R, Towers AL, Morichi V, et al. A manual of guidelines to score the modified cumulative illness rating scale and its validation in acute hospitalized elderly patients. J Am Geriatr Soc. 2008;56(10):1926-31.

40. Clements RM. Reducing psychotropic medications in elderly rehabilitation inpatients with a fall-related admission: how often is it happening? Geriatr Gerontol Int. 2008;8(3):139-42.

41. Wilson MG, Lee TC, Hass A, Tannenbaum C, McDonald EG. EMPOWERing Hospitalized Older Adults to Deprescribe Sedative Hypnotics: A Pilot Study. J Am Geriatr Soc. 2018;66(6):1186-9.

42. Scott S, Clark A, Farrow C, May H, Patel M, Twigg MJ, et al. Deprescribing admission medication at a UK teaching hospital; a report on quantity and nature of activity. Int J Clin Pharm. 2018;40(5):991-6.

43. Baandrup L, Ebdrup BH, Rasmussen J, Lindschou J, Gluud C, Glenthøj BY. Pharmacological interventions for benzodiazepine discontinuation in chronic benzodiazepine users. Cochrane Database Syst Rev. 2018;3(3):Cd011481.

44. Martin P, Tamblyn R, Benedetti A, Ahmed S, Tannenbaum C. Effect of a Pharmacist-Led Educational Intervention on Inappropriate Medication Prescriptions in Older Adults: The D-PRESCRIBE Randomized Clinical Trial. JAMA. 2018;320(18):1889-98.

45. Ailabouni N, Mangin D, Nishtala PS. DEFEAT-polypharmacy: deprescribing anticholinergic and sedative medicines feasibility trial in residential aged care facilities. Int J Clin Pharm. 2019;41(1):167-78.
46. Ammerman CA, Simpkins BA, Warman N, Downs TN. Potentially Inappropriate Medications in Older Adults: Deprescribing with a Clinical Pharmacist. J Am Geriatr Soc. 2019;67(1):115-8.

47. Walsh KA, O'Riordan D, Kearney PM, Timmons S, Byrne S. Improving the appropriateness of prescribing in older patients: a systematic review and meta-analysis of pharmacists' interventions in secondary care. Age Ageing. 2016;45(2):201-9.

48. Carr F, Tian P, Chow J, Guzak J, Triscott J, Mathura P, et al. Deprescribing benzodiazepines among hospitalised older adults: quality improvement initiative. BMJ Open Qual. 2019;8(3):e000539.

49. Neville HL, Losier M, Pitman J, Gehrig M, Isenor JE, Minard LV, et al. Point Prevalence Survey of Benzodiazepine and Sedative-Hypnotic Drug Use in Hospitalized Adult Patients. Canadian J Hosp Pharmacy. 2020;73(3):193-201.

50. Wedmann F, Himmel W, Nau R. Medication and medical diagnosis as risk factors for falls in older hospitalized patients. Eur J Clin Pharmacol. 2019;75(8):1117-24.

51. Fagnoni $P$, Limat $S$, Haffen $E$, Henon $T$, Jacquet M, Sechter $D$, et al. Does hospitalisation affect hypnotic and anxiolytic drug prescribing? Pharm World Sci. 2007;29(6):611-8.

52. Pottie K, Thompson W, Davies S, Grenier J, Sadowski CA, Welch V, et al. Deprescribing benzodiazepine receptor agonists. Evid Based Clin Pract Guideline. 2018;64(5):339-51.

53. Goyal P, Anderson TS, Bernacki GM, Marcum ZA, Orkaby AR, Kim D, et al. Physician Perspectives on Deprescribing Cardiovascular Medications for Older Adults. J Am Geriatr Soc. 2020;68(1):78-86.

54. Scott $S$, Wright DJ, Bhattacharya D. The role of behavioural science in changing deprescribing practice. Br J Clin Pharmacol. 2021;87(1):39-41.

55. Bayliss EA, Shetterly SM, Drace ML, Norton J, Green AR, Reeve E, et al. The OPTIMIZE patient- and family-centered, primary care-based deprescribing intervention for older adults with dementia or mild cognitive impairment and multiple chronic conditions: study protocol for a pragmatic cluster randomized controlled trial. Trials. 2020;21(1):542.

56. Gnjidic D, Ong HMM, Leung C, Jansen J, Reeve E. The impact of in hospital patient-education intervention on older people's attitudes and intention to have their benzodiazepines deprescribed: a feasibility study. Therapeutic Adv Drug Saf. 2019;10:2042098618816562.

\section{Publisher's Note}

Springer Nature remains neutral with regard to jurisdictional claims in published maps and institutional affiliations.

\footnotetext{
Ready to submit your research? Choose BMC and benefit from:

- fast, convenient online submission

- thorough peer review by experienced researchers in your field

- rapid publication on acceptance

- support for research data, including large and complex data types

- gold Open Access which fosters wider collaboration and increased citations

- maximum visibility for your research: over $100 \mathrm{M}$ website views per year
}

At BMC, research is always in progress.

Learn more biomedcentral.com/submissions 